\title{
Lacunary Interpolation at Odd and Even Nodes
}

\author{
Kulbhushan Singh \\ Department of Mathematics \\ and Computer Science \\ The Papua New Guinea \\ University of Technology, \\ Lae, Papua New Guinea
}

\author{
Ambrish Kumar Pandey \\ Department of Mathematics \\ Integral University, \\ Lucknow, India
}

\begin{abstract}
Here a special $(0,2 ; 0,3)$ lacunary interpolation scheme is considered where the data are prescribed unevenly at even and odd nodes of an arbitrarily defined partition of the unit interval $\quad I=[0,1]$.

The problem described as, we have the function values and second derivatives at odd nodes, whereas function values and the thirdderivatives ateven nodes are known, weproved that there existsa unique quantic spline of continuity class $C^{2}$ by solving the above mentioned interpolation scheme.
\end{abstract}

Furthermore, it is also proved that this spline function converges to the given function with the desired order of accuracy.

\section{Keywords}

Lacunary interpolation, splines.

\section{INTRODUCTION}

In this paper we have discussed $(0,2 ; 0,3)$ lacunary interpolation problem where the function value is prescribed at every node of the partitionof the unit interval whereas third and second derivatives are prescribed alternately at even and odd nodes.

For more related workone is referred to [1] - [6].Let us denotes by $S_{n, 5}^{3}$, the class of quintic splines $s(x)$ on the unit interval $[0,1]$ such that

(i) $\mathrm{s}(\mathrm{x}) \in \mathrm{C} 3[0,1]$

(ii) $\mathrm{s}(\mathrm{x}) \in \pi 5$ on each $[\mathrm{v} / \mathrm{n},(\mathrm{v}+1) / \mathrm{n}], 0 \leq \mathrm{v} \leq \mathrm{n}-1$.

We shall prove the following theorems.

Theorem 1

Foreveryodd integer $n$ and for every given set of $2 n+4$ real numbersf $f_{0}, \mathrm{f}_{1}, \ldots, \mathrm{f}_{\mathrm{n}} ; \mathrm{f}_{0}^{\prime \prime \prime}, \mathrm{f}_{2}^{\prime \prime \prime}, \ldots, \mathrm{f}_{\mathrm{n}-1}^{\prime \prime \prime} ; \mathrm{f}_{1}^{\prime \prime}, \mathrm{f}_{3}^{\prime \prime}, \ldots, \mathrm{f}_{\mathrm{n}}^{\prime \prime} ; \mathrm{f}_{0}^{\prime \prime} ;$ $\mathrm{f}_{\mathrm{n}}^{\prime}$ there exists a unique spline $\mathrm{s}(\mathrm{x}) \in \mathrm{Sn}, 5(3)$ such that
(1.1) $\mathrm{s}(\mathrm{v} / \mathrm{n})=\mathrm{f}_{v}$; $\mathrm{v}=0,1, \ldots, \mathrm{n}$
(1.2) $\mathrm{s}^{\prime \prime \prime}(2 \mathrm{v} / \mathrm{n})=\mathrm{f}_{2 v}^{\prime \prime \prime}$; $\mathrm{v}=0,1, \ldots,(\mathrm{n}-1) / 2$,
(1.3) $\mathrm{s}^{\prime \prime}((2 \mathrm{v}+1) / \mathrm{n})=\mathrm{f}_{2 v+1}^{\prime \prime} ; \mathrm{v}=0,1, \ldots,(\mathrm{n}-1) / 2$.
(1.4) $\mathrm{s}^{\prime}(0)=\mathrm{f}_{0}, \quad \mathrm{~s}^{\prime}(1)=\mathrm{f}_{\mathrm{n}}^{\prime}$

\section{THEOREM2}

Let $\mathrm{f} \in \mathrm{C} 4[0,1]$ and $\mathrm{n}$ be an odd integer. Then for the unique quintic spline $\operatorname{Sn}(\mathrm{x})$ satisfying conditions of Theorem1 with

$f_{v}=f(v / n), \quad v=0,1, \ldots, \mathrm{n}$,

$f_{2 v}^{\prime \prime \prime}=f^{\prime \prime \prime}(2 v / n), v=0,1, \ldots,(\mathrm{n}-1) / 2$,

$f_{2 v+1}^{\prime \prime}=f^{\prime \prime}(2 v+1 / n), v=0,1, \ldots,(\mathrm{n}-1) / 2$,

$f_{0}^{\prime}=f^{\prime}(0)$ and $f_{n}^{\prime}=f^{\prime}(1)$;

We have

$$
\left\|S_{n}{ }^{(r)}(x)-f^{(r)}(x)\right\|_{\infty} \leq K_{v} n^{r-3} \omega_{4}(1 / n)+2 n^{r-4}\left\|f^{(4)}\right\|_{\infty} \text {. }
$$

Here Kv are different constants depending on $\mathrm{v}$ and $\omega 4($.)denotes the modulus of continuity of $f(4)$.

\section{PRELIMINARIES}

It can be verified that if $\mathrm{P}(\mathrm{x})$ is a quantic on $[0,1]$ then

(3.1) $\mathrm{P}(\mathrm{x})=\mathrm{P}(0) \mathrm{A} 0(\mathrm{x})+. \mathrm{P}(1) \mathrm{A} 1(\mathrm{x})+\mathrm{P}^{\prime \prime}(0) \mathrm{A} 2(\mathrm{x})+$

$$
\mathrm{P}^{\prime \prime}(1) \mathrm{A} 3(\mathrm{x})+\mathrm{P}(4)(0) \mathrm{A} 4(\mathrm{x})+\mathrm{P}(4)(1) \mathrm{A} 5(\mathrm{x}) \text {. }
$$

Where

$$
\begin{aligned}
& \mathrm{A}_{0}(\mathrm{x})=\mathrm{x} \\
& \mathrm{A}_{1}(\mathrm{x})=1 / 6\left(\mathrm{x}^{3}-3 \mathrm{x}^{2}+2 \mathrm{x}\right) \\
& \mathrm{A}_{2}(\mathrm{x})=1 / 2\left(\mathrm{x}^{2}-\mathrm{x}\right) \\
& \mathrm{A}_{3}(\mathrm{x})=-1 / 120\left(\mathrm{x}^{5}-5 \mathrm{x}^{4}+20 \mathrm{x}^{2}-16 \mathrm{x}\right) \\
& \mathrm{A}_{4}(\mathrm{x})=1 / 120\left(\mathrm{x}^{5}-10 \mathrm{x}^{2}+9 \mathrm{x}\right) \\
& \mathrm{A}_{5}(\mathrm{x})=1 / 120\left(2 \mathrm{x}^{5}+\mathrm{x}^{2}+2 \mathrm{x}\right)
\end{aligned}
$$

A quintic $\mathrm{Q}(\mathrm{x})$ on $[1,2]$ can be expressed as

(3.2) $\mathrm{Q}(\mathrm{x})=\mathrm{Q}(2) \mathrm{A} 0(2-\mathrm{x})+\mathrm{Q}(1) \mathrm{A} 1(2-\mathrm{x})+$

$$
\begin{aligned}
& +\mathrm{Q}^{\prime}(2) \mathrm{A} 2(2-\mathrm{x})+\mathrm{Q}^{\prime \prime} \text { (1) } \mathrm{A} 3(2-\mathrm{x})+ \\
& +\mathrm{Q}^{\prime \prime} \text { (2) } \mathrm{A} 4(2-\mathrm{x})+\mathrm{Q}^{\prime \prime} \text { (1) } \mathrm{A} 5(2-\mathrm{x})
\end{aligned}
$$

For later reference we note that 


$\left\{\begin{array}{ccccc}A_{0}^{\prime}(0)=1, & A_{0}^{\prime \prime}(0)=0, & A_{0}^{\prime \prime \prime}(0)=0, & A_{0}{ }^{(4)}(0)=0, & A_{0}{ }^{(5)}(0)=0, \\ A_{0}^{\prime}(1)=1, & A_{0}^{\prime \prime}(1)=0, & A_{0}^{\prime \prime \prime}(1)=0, & A_{0}{ }^{(4)}(1)=0, & A_{0}{ }^{(5)}(1)=0, \\ A_{1}^{\prime}(0)=1 / 3, & A_{1}^{\prime \prime}(0)=-1, & A_{1}^{\prime \prime \prime}(0)=1, & A_{1}{ }^{(4)}(0)=0, & A_{1}{ }^{(5)}(0)=0, \\ A_{1}^{\prime}(1)=-1 / 6, & A_{1}^{\prime \prime}(1)=0, & A_{1}^{\prime \prime \prime}(1)=1, & A_{1}{ }^{(4)}(1)=0, & A_{1}{ }^{(5)}(1)=0, \\ A_{2}^{\prime}(0)=1 / 2, & A_{2}^{\prime \prime}(0)=-1, & A_{2}^{\prime \prime \prime}(0)=0, & A_{2}{ }^{(4)}(0)=0, & A_{2}{ }^{(5)}(0)=0, \\ A_{2}^{\prime}(1)=-1 / 2, & A_{2}^{\prime \prime}(0)=1, & A_{2}^{\prime \prime \prime}(1)=0, & A_{2}{ }^{(4)}(1)=0, & A_{2}{ }^{(5)}(1)=0, \\ A_{3}^{\prime}(0)=2 / 15, & A_{3}^{\prime \prime}(0)=-1 / 3, & A_{3}^{\prime \prime \prime}(0)=0, & A_{3}{ }^{(4)}(0)=1 / 5, & A_{3}{ }^{(5)}(0)=-1, \\ A_{3}^{\prime}(1)=-3 / 40, & A_{3}^{\prime \prime}(0)=0, & A_{3}^{\prime \prime \prime}(1)=1 / 2, & A_{3}{ }^{(4)}(1)=-4 / 5, & A_{3}{ }^{(5)}(1)=-1, \\ A_{4}^{\prime}(0)=3 / 40, & A_{4}^{\prime \prime}(0)=-1 / 6, & A_{4}^{\prime \prime \prime}(0)=0, & A_{4}{ }^{(4)}(0)=0, & A_{4}{ }^{(5)}(0)=1, \\ A_{4}^{\prime}(1)=-1 / 20, & A_{4}^{\prime \prime}(1)=0, & A_{4}^{\prime \prime \prime}(1)=-1 / 2, & A_{4}{ }^{(4)}(1)=0, & A_{4}{ }^{(5)}(1)=1\end{array}\right.$

A quintic $\mathrm{P}(\mathrm{x})$ in $[0,1]$ can be expressed in the following form.

(3.4) $\mathrm{P}(\mathrm{x})=\mathrm{P}(0) \mathrm{B} 0(\mathrm{x})+\mathrm{P}(1) \mathrm{B} 1(\mathrm{x})+\mathrm{P}^{\prime}{ }_{(0) \mathrm{B} 2(\mathrm{x})+\mathrm{P}^{\prime}}{ }_{(1)}$ $\mathrm{B} 3(\mathrm{x})+\mathrm{P}^{\prime \prime \prime}(0) \mathrm{B} 4(\mathrm{x})+\mathrm{P}^{\prime \prime}$ (1) B5(x),

Where $\quad \begin{aligned} \mathrm{B}_{0}(\mathrm{x}) & =1 / 3\left(-8 \mathrm{x}^{5}+15 \mathrm{x}^{4}-10 \mathrm{x}^{2}+3\right), \\ \mathrm{B}_{1}(\mathrm{x}) & =1 / 3\left(8 \mathrm{x}^{5}-15 \mathrm{x}^{4}+10 \mathrm{x}^{2}\right), \\ \mathrm{B}_{2}(\mathrm{x}) & =-\mathrm{x}^{5}+2 \mathrm{x}^{4}-2 \mathrm{x}^{2}+\mathrm{x}, \\ \mathrm{B}_{3}(\mathrm{x}) & =1 / 3\left(-5 \mathrm{x}^{5}+9 \mathrm{x}^{4}-4 \mathrm{x}^{2}\right),\end{aligned}$
$\mathrm{B}_{4}(\mathrm{x})=1 / 18\left(\mathrm{x}^{5}-3 \mathrm{x}^{4}+3 \mathrm{x}^{3}-\mathrm{x}^{2}\right)$

$B_{5}(x)=1 / 6\left(2 x^{5}-3 x^{4}+x^{2}\right)$,
Also a quintic $\mathrm{Q}(\mathrm{x})$ in $[1,2]$ can be written as (3.5) $\mathrm{Q}(\mathrm{x})=\mathrm{Q}(2) \mathrm{B}_{0}(2-\mathrm{x})+\mathrm{Q}(1) \mathrm{B}_{1}(2-\mathrm{x})-\mathrm{Q}^{\prime}(2) \mathrm{B}_{2}(2-$ $\mathrm{x})-$

$$
\mathrm{Q}^{\prime}(1) \mathrm{B}_{3}(2-\mathrm{x})-\mathrm{Q}^{\prime \prime \prime}(2) \mathrm{B}_{4}(2-\mathrm{x})+\mathrm{Q}^{\prime \prime}(1) \mathrm{B}_{5}(2-
$$

$\mathrm{x})$.

For later reference we have

$$
(3.6)\left\{\begin{array}{llll}
B_{0}^{\prime}(0)=0, & B_{0}^{\prime \prime}(0)=-20 / 3, & B_{0}^{\prime \prime \prime}(0)=120, & B_{0}{ }^{(4)}(0)=120, \\
B_{0}^{\prime}(1)=0, & B_{0}^{\prime \prime}(1)=0, & B_{0}^{\prime \prime \prime}(1)=-40, & B_{0}{ }^{(4)}(1)=-200, \\
B_{1}^{\prime}(0)=0, & B_{1}^{\prime \prime}(0)=20 / 3, & B_{1}^{\prime \prime \prime}(0)=-120, & B_{1}{ }^{(4)}(0)=-120, \\
B_{1}^{\prime}(1)=0, & B_{1}^{\prime \prime}(1)=0, & B_{1}^{\prime \prime \prime}(1)=40, & B_{1}{ }^{(4)}(1)=200, \\
B_{2}^{\prime}(0)=1, & B_{2}^{\prime \prime}(0)=0, & B_{2}^{\prime \prime \prime}(0)=0, & B_{2}{ }^{(4)}(0)=48, \\
B_{2}^{\prime}(1)=0, & B_{2}^{\prime \prime}(1)=0 & B_{2}^{\prime \prime \prime}(1)=-12, & B_{2}{ }^{(4)}(1)=-72, \\
B_{3}^{\prime}(0)=0, & B_{3}^{\prime \prime}(0)=-8 / 3 & B_{3}^{\prime \prime \prime}(0)=0, & B_{3}{ }^{(4)}(0)=72, \\
B_{3}^{\prime}(1)=1, & B_{3}^{\prime \prime}(1)=0, & B_{3}^{\prime \prime \prime}(1)=-28, & B_{3}{ }^{(4)}(1)=-128, \\
B_{4}^{\prime}(0)=0, & B_{4}^{\prime \prime}(0)=-1 / 9, & B_{4}^{\prime \prime \prime}(0)=1, & B_{4}{ }^{(4)}(0)=-4, \\
B_{4}^{\prime}(1)=0, & B_{4}^{\prime \prime}(1)=0, & B_{4}^{\prime \prime \prime}(1)=1 / 3, & B_{4}{ }^{(4)}(1)=8 / 3, \\
B_{5}^{\prime}(0)=0 & B_{5}^{\prime \prime}(0)=1 / 3 & B_{5}^{\prime \prime \prime}(0)=0 & B_{5}{ }^{(4)}(1)=-12 \\
B_{5}^{\prime}(1)=0 & B_{5}^{\prime \prime}(1)=1 & B_{5}^{\prime \prime \prime}(1)=8 & B_{5}{ }^{(4)}(1)=28
\end{array}\right.
$$

(3.7) $\mathrm{P}(0)=-20 / 3 \mathrm{P}(0)+20 / 3 \mathrm{P}(1)-4 \mathrm{P}(0)-8 / 3 \mathrm{P}$ (1) $-1 / 9$ P "' (0) +1/3 P" (1),

(3.8) $\mathrm{P}^{\prime \prime}(1)=-40 \mathrm{P}(0)+40 \mathrm{P}(1)-12 \mathrm{P}^{\prime}(0)-28 \mathrm{P}^{\prime}(1)+$ $+1 / 3 \mathrm{P}^{\prime \prime}(0)+8 \mathrm{P}^{\prime \prime}(1)$,

(3.9) $\mathrm{P}^{(4)}(0)=120 \mathrm{P}(0)-120 \mathrm{P}(1)+48 \mathrm{P}^{\prime}(0)+72 \mathrm{P}^{\prime}(1)-$
(3.10) $\mathrm{P}^{(4)}(1)=-200 \mathrm{P}(0)+200 \mathrm{P}(1)-72 \mathrm{P}(0)-128 \mathrm{P}^{\prime}(1)+$

$$
+8 / 3 \mathrm{P}^{\prime \prime}(0)+28 \mathrm{P} "(1)
$$

Similarly from equation (3.5) and (3.6) we get (3.11) $\mathrm{Q}^{\prime \prime}(2)=-20 / 3 \mathrm{Q}(2)+20 / 3 \mathrm{Q}(1)+4 \mathrm{Q}^{\prime}(2)+8 / 3 \mathrm{Q}^{\prime}(1)$ $+$ +1/9 Q "' (2)+1/3 Q" (1), 
(3.12) Q '" $(1)=40 \mathrm{Q}(2)-40 \mathrm{Q}(1)-12 \mathrm{Q}^{\prime}(2)-28 \mathrm{Q}(1)+$ +1/3 Q "' (2) - 8 Q" (1),

(3.13) $\mathrm{Q}^{(4)}(2)=120 \mathrm{Q}(2)-120 \mathrm{Q}(1)-48 \mathrm{Q}^{\prime}(2)-72 \mathrm{Q}^{\prime}(1)$

$$
\text { +4 Q"' (2) - } 12 \mathrm{Q}^{\prime \prime}(1)
$$

(3.14) $\mathrm{Q}^{(4)}(1)=-200 \mathrm{Q}(2)+200 \mathrm{Q}(1)+72 \mathrm{Q}^{\prime}(2)+$

$$
\text { +128 Q' (1)-8/3 Q "' (2)+28 Q" (1), }
$$

\section{Proof of Theorem1:}

For a given $\mathrm{s}(\mathrm{x}) \in \mathrm{S}_{\mathrm{n}, 5}{ }^{(3)}$ set $\mathrm{h}=1 / \mathrm{n}$ and

$\mathrm{M}_{v}=\mathrm{s}^{(4)}(v \mathrm{~h}+), \quad v=0,1, \ldots, \mathrm{n}-1$,

$\mathrm{N}_{v}=\mathrm{s}^{(4)}(v \mathrm{~h}-), \quad v=0,1, \ldots, \mathrm{n}$.

Since $\mathrm{S}^{(4)}(\mathrm{x})$ is linear in each interval $[v \mathrm{~h},(v+1) \mathrm{h}]$, it is completely determined by the $2 \mathrm{n}$ constants $\left\{M_{\nu}\right\}^{n-1}$ and $\left\{N_{v}\right\}_{1}^{n}$. Also if $\mathrm{s}(\mathrm{x})$ satisfies the requirements of Theorem 1, it follows from equation $(1.1)-(1.3)$ and $(3.1)-(3.2)$ that for

$2 v \mathrm{~h} \leq \mathrm{x} \leq(2 v+1) \mathrm{h}, v=0,1, \ldots,(\mathrm{n}-1) / 2$, it must have the form

$(1.5) \mathrm{s}(\mathrm{x})=\quad \mathrm{f}_{2 v} \quad \mathrm{~A}_{0}\left(\frac{(2 v+1) h-x}{h}\right)+\mathrm{f}_{2 v+1} \quad \mathrm{~A}_{0}$ $\left(\frac{\mathrm{x}-2 \mathrm{vh})}{\mathrm{h}}\right)$

$+\mathrm{h}^{3} f_{2 v}^{\prime \prime} \mathrm{A}_{1} \frac{(\mathrm{x}-2 \mathrm{vh})}{\mathrm{h}}+\mathrm{h}^{2} f_{2 v+1}^{\prime \prime} \mathrm{A}_{2} \frac{(x-2 v h)}{h}+$

$+\mathrm{h}^{4} \mathrm{M}_{2 v} \mathrm{~A}_{3} \frac{(\mathrm{X}-2 \mathrm{vh})}{\mathrm{h}}+\mathrm{h}^{4} \mathrm{~N}_{2 v+1} \mathrm{~A}_{4} \frac{(x-2 v h)}{h}$

For $(2 v+1) \mathrm{h} \leq \mathrm{x} \leq(2 v+2) \mathrm{h}, \quad v=0,1, \ldots,(\mathrm{n}-3) / 2$

(1.6) $\mathrm{s}(\mathrm{x})=\mathrm{f}_{2 v+1} \mathrm{~A}_{0}\left(\frac{(2 v+2) h-x}{h}\right)+\mathrm{f}_{2 v+2} \mathrm{~A}_{0}$ $\left(\frac{x-(2 v+1) h}{h}\right)-\mathrm{h}^{3} f_{2 v+2}^{\prime \prime} \mathrm{A}_{1}\left(\frac{(2 v+2) h-x}{h}\right)+$

$+\mathrm{h}^{2} f_{2 v+1}^{\prime \prime} \mathrm{A}_{2}\left(\frac{(2 \mathrm{v}+2) \mathrm{h}-\mathrm{x}}{\mathrm{h}}\right)+$

$+\mathrm{h}^{4} \mathrm{~N}_{2 v+2} \mathrm{~A}_{3}\left(\frac{(2 v-2) h-x}{h}\right)+\mathrm{h}^{4}$

$\mathrm{M}_{2 v+1} \mathrm{~A}_{4}\left(\frac{(2 \mathrm{v}+2) \mathrm{h}-\mathrm{x}}{\mathrm{h}}\right)$

Since we have $\mathrm{s}^{\prime}(0)=\mathrm{f}_{0}^{\prime}$; therefore equation (1.1) implies

$$
16 M_{0}+9 N_{1}=120 h^{-4}\left[f_{0}-f_{1}+h f_{0}^{\prime}+h^{2}\right.
$$

$\left./ 2 \mathrm{f}_{1}^{\prime \prime}-\mathrm{h}^{3} \mathrm{f}_{0}^{\prime \prime}\right]$

Similarly using condition $s^{\prime}(1)=f_{0}^{\prime}$ we have from equation (1.6) $3 M_{n-1}+2 N_{n}=40 h^{-4}\left[-f_{n-1}{ }^{\prime}+f^{\prime \prime}{ }_{n}-h f_{n}{ }^{\prime}\right.$ $\left.+h^{2} / 2 f_{n} "-h^{3} / 3 f_{n-1} " '\right]$.

Also using $\quad \mathrm{s}^{\prime}((2 v+1) / \mathrm{h}-)=\mathrm{s}^{\prime}((2 \mathrm{v}+1) / \mathrm{h}+)$

and $\mathrm{s}^{\prime \prime}((2 v+1) / \mathrm{h}-)=\mathrm{s} \quad((2 v+1) / \mathrm{h}+)$, we get

(1.9) $3\left(\mathrm{M}_{2 v}+\mathrm{N}_{2 v+2}\right)+\underset{\prime \prime \prime}{2}\left(\mathrm{M}_{2 v+1}+\mathrm{N}_{2 \mathrm{v}+1}\right)=-40^{-4}\left[\mathrm{f}_{2 v}-2 \mathrm{f}_{2 v+1}+\right.$ $\left.\mathrm{f}_{2 v+2}+\mathrm{h}^{2} f_{2 v+1}^{\prime \prime}-\mathrm{h}^{3} / 6\left(f_{2 v}^{\prime \prime \prime}-f_{2 v+2}^{\prime \prime \prime}\right)\right]$

and

$(1.10) \quad\left(\mathrm{M}_{2 v}+\mathrm{N}_{2 v+2}\right)+\left(\mathrm{M}_{2 v+1}+\mathrm{N}_{2 v+1}\right)=-2 \mathrm{~h}^{-1}$ $\left(f_{2 v}^{\prime \prime \prime}-f_{2 v+2}^{\prime \prime \prime}\right)$

Similarly froms $((2 v+2) / \mathrm{h}-)=\mathrm{s}^{\prime}((2 v+2) / \mathrm{h}+)$

and $\mathrm{s}^{\prime \prime}((2 v+2) / \mathrm{h}-)=\mathrm{s}^{\prime \prime}((2 v+2) / \mathrm{h}+)$, we get

(1.11) $16\left(\mathrm{M}_{2 v+1}+\mathrm{N}_{2 v+2}\right)+9\left(\mathrm{M}_{2 v+1}+\mathrm{N}_{2 v+3}\right)$

$-120 \mathrm{~h}^{-4}\left[\mathrm{f}_{2 v+2}-2 \mathrm{f}_{2 v+2}+\mathrm{f}_{2 v+3}-\mathrm{h}^{2} / 2\left(f_{2 v+1}^{\prime \prime}+f_{2 v+3}^{\prime \prime}\right)\right]$

and

(1.12) $\quad 2\left(\mathrm{M}_{2 v+2}-\mathrm{N}_{2 v+2}\right)-\left(\mathrm{M}_{2 v+1}+\mathrm{N}_{2 v+3}\right)=6 \mathrm{~h}^{-4}$ $\left[\mathrm{h}^{2}\left(f_{2 v+3}^{\prime \prime}-f_{2 v+1}^{\prime \prime}\right)-2 \mathrm{~h}^{3} f_{2 v+2}^{\prime \prime \prime}\right]$

The constants $\left\{M_{v}\right\}_{0}^{n-1}$ and $\left\{N_{v}\right\}_{1}^{n}$ will be determined uniquely from equations (1.7) to (1.12) if the corresponding homogeneous system of equations given by

$$
\begin{aligned}
& 16 \mathrm{M}_{0}+9 \mathrm{~N}_{1}=0, \\
& 3 \mathrm{M}_{n-1}+2 \mathrm{~N}_{\mathrm{n}}=0, \\
& 3\left(\mathrm{M}_{2 v}+\mathrm{N}_{2 v+2}\right)+2\left(\mathrm{M}_{2 v+1}+\mathrm{N}_{2 v+1}\right)=0, \\
& \left(\mathrm{M}_{2 v}+\mathrm{N}_{2 v+2}\right)+2\left(\mathrm{M}_{2 v+1}+\mathrm{N}_{2 v+1}\right)=0, \\
& 16\left(\mathrm{M}_{2 v+2}+\mathrm{N}_{2 v+2}\right)+9\left(\mathrm{M}_{2 v+1}+\mathrm{N}_{2 v+3}\right)=0, \\
& 2\left(\mathrm{M}_{2 v+2}-\mathrm{N}_{2 v+2}\right)-\left(\mathrm{M}_{2 v+1}-\mathrm{N}_{2 v+3}\right)=0,
\end{aligned}
$$

has only the zero solution.Now from equation (1.15) and (1.16) we have

$$
\begin{aligned}
& \mathrm{M}_{2 v}+\mathrm{N}_{2 v+2}=0 \\
& \mathrm{M}_{2 v+1}+\mathrm{N}_{2 v+1}=0 .
\end{aligned}
$$

Putting $\mathrm{M}_{2 \mathrm{v}+1}=-\mathrm{N}_{2 \mathrm{v}+1}$ and $\mathrm{N}_{2 \mathrm{v}+2}=-\mathrm{M}_{2 \mathrm{v}}$ from equation (1.19) in equation (1.17) we have

$$
16\left(\mathrm{M}_{2 \mathrm{v}+2}-\mathrm{N}_{2 \mathrm{v}}\right)+9\left(\mathrm{~N}_{2 \mathrm{v}+3}-\mathrm{N}_{2 \mathrm{v}+1}\right)=0
$$

Taking sum for $\mathrm{v}=0,1, \ldots,(\mathrm{n}-3) / 2$, we get

$$
16\left(M_{n-1}-M_{0}\right)+9\left(N_{n}-N_{1}\right)=0,
$$

or

$$
16\left(\mathrm{M}_{0}-\mathrm{M}_{\mathrm{n}-1}\right)+9\left(\mathrm{~N}_{1}-\mathrm{N}_{\mathrm{n}}\right)=0,
$$

Using equation (1.13) we obtain

$$
16 \mathrm{M}_{\mathrm{n}-1}+9 \mathrm{~N}_{\mathrm{n}}=0,
$$

This gives, on using equation (1.13) $\mathrm{M}_{\mathrm{n}-1}=0, \mathrm{~N}_{\mathrm{n}}=0$, 
Then putting $\mathrm{v}=(\mathrm{n}-3) / 2$ and $\mathrm{M}_{\mathrm{n}-1}=0 \quad \mathrm{~N}_{\mathrm{n}}=0$ in equations (1.17) and (1.18) we have

$$
16 \mathrm{~N}_{\mathrm{n}-1}+9 \mathrm{M}_{\mathrm{n}-2}=0
$$

and

$$
2 \mathrm{~N}_{\mathrm{n}-1}+\mathrm{M}_{\mathrm{n}-2}=0
$$

From these two equations we get

$$
\mathrm{M}_{\mathrm{n}-2}=0, \mathrm{~N}_{\mathrm{n}-1}=0
$$

Following similar arguments for different values of $\mathrm{v}$, we have

$$
\begin{array}{ll}
M_{n-3}=0, & N_{n-2}=0 \\
M_{n-4}=0, & N_{n-3}=0 ; \\
\ldots & \\
\cdots & \\
M_{2}=0, & N_{3}=0 ; \\
M_{1}=0, & N_{2}=0 ; \\
M_{0}=0, & N_{1}=0 ;
\end{array}
$$

Therefore

$\mathrm{M}_{0}=\mathrm{M}_{1}=\ldots=\mathrm{M}_{\mathrm{n}-1}=0$

and

$\mathrm{N}_{1}=\mathrm{N}_{2}=\ldots=\mathrm{N}_{\mathrm{n}}=0$.

This completes the proof of Theorem1.

For the proof of Theorem 2 we need to prove the following two lemmas.

\section{Lemma 1}

Let $\mathrm{f} \in \mathrm{C}^{4}[0,1], \mathrm{n}$ any odd integer and $\mathrm{h}=1 / \mathrm{n}$. Then for the unique spline $\mathrm{s}_{\mathrm{n}}(\mathrm{x})$ Theorem (1),

we have

$$
\begin{aligned}
& \left|\mathrm{A}_{2 v}\right|=\left|\mathrm{s}_{\mathrm{n}}{ }^{\prime}(2 v \mathrm{~h})-\mathrm{f}^{\prime}(2 v \mathrm{~h})\right| \\
& \leq 41 \mathrm{~h}^{2} / 18 \omega_{4}(\mathrm{~h}), \quad v=0,1, \ldots,(\mathrm{n}-1) / 2
\end{aligned}
$$

and

$$
\begin{aligned}
& \left.\left|\mathrm{A}_{2 v+1}\right|=\mid \mathrm{s}_{\mathrm{n}}{ }^{\prime} \quad((2 v+1) \mathrm{h})-\mathrm{f}^{\prime}(2 v+1) \mathrm{h}\right) \mid \\
& \leq \mathrm{C}_{v} \mathrm{~h}^{2} \omega_{4}(\mathrm{~h}), \quad v=0,1, \ldots,(\mathrm{n}-1) / 2
\end{aligned}
$$

where $\mathrm{C}_{v}$ are the different constants depending on $v$.

\section{Proof}

From equation (P.8) we have for $2 v \mathrm{~h} \leq \mathrm{x} \leq(2 v+1) \mathrm{h}$

$$
h^{3} s^{\prime \prime}((2 v+1) h)=-40 f_{2 v}+40 f_{2 v+1}-12 h
$$

s $(2 \mathrm{vh})-$

$-28 \mathrm{~h} \mathrm{~s}^{\prime}((2 \mathrm{v}+1) \mathrm{h})+\mathrm{h}^{3} / 3 \mathrm{f}_{2 \mathrm{v}}{ }^{\prime \prime}+8 \mathrm{~h}^{2} \mathrm{f}_{2 \mathrm{v}+1}{ }^{\prime \prime}$

Similarly for

$(2 \mathrm{v}+1) \mathrm{h} \leq \mathrm{x} \leq(2 \mathrm{v}+2) \mathrm{h}$, we have

(L.4)

$$
h^{3} s^{\prime \prime}((2 v+1) h)=40 f_{2 v+2}+40 f_{2 v+1}-12
$$

h s' $(2 v+2) h)$

$-28 \mathrm{~h} \mathrm{~s}^{\prime}((2 \mathrm{v}+1) \mathrm{h})+\mathrm{h}^{3} / 3 \mathrm{f}_{2 \mathrm{v}+2}{ }^{\prime \prime}-8 \mathrm{~h}^{2} \mathrm{f}_{2 \mathrm{v}+1}$ dó
Subtracting (1.8) from (L.3) we get

$$
12\left(A_{2 v}-A_{2 v+2}\right)=-40\left(f_{2 v}+f_{2 v+2}\right)+80
$$

$\mathrm{f}_{2 \mathrm{v}+1}+16 \mathrm{~h}^{2} \mathrm{f}_{2 \mathrm{v}+1}$

$+\mathrm{h}^{3} / 3\left(\mathrm{f}_{2 \mathrm{v}}{ }^{\prime \prime}-\mathrm{f}_{2 \mathrm{v}+2}{ }^{\prime \prime}\right)+12 \mathrm{~h}\left(\mathrm{f}_{2 \mathrm{v}+1}{ }^{\prime}-\mathrm{f}_{2 \mathrm{v}}{ }^{\prime}\right)$.

Using Taylor expansion for the function $\mathrm{f}$ and its derivatives on R.H. S., we get

$12\left(\mathrm{~A}_{2 \mathrm{v}}-\mathrm{A}_{2 \mathrm{v}+2}\right)=\mathrm{h}^{3}\left[-80 / 3 \mathrm{f}^{(4)}(\gamma)+10 / 3 \mathrm{f}^{(4)}(\alpha)+24 / 3 \mathrm{f}^{(4)}(\beta)\right.$

$\left.-2 / 3 \mathrm{f}^{(4)}(\delta)+45 / 3 \mathrm{f}^{(4)}(\eta)\right]$

$2 \mathrm{vh} \leq \alpha, \beta \leq(2 \mathrm{v}+1) \mathrm{h} ;(2 \mathrm{v}+1) \mathrm{h} \leq \gamma, \delta, \eta \leq(2 \mathrm{v}+2) \mathrm{h}$.

Fixing $\mathrm{k}, 0 \leq \mathrm{k} \leq(\mathrm{n}-2) / 2$, and summing both sides of the above equation for

$\mathrm{v}=\mathrm{k}, \mathrm{k}+1, \ldots \ldots,(\mathrm{n}-2) / 2$, we have

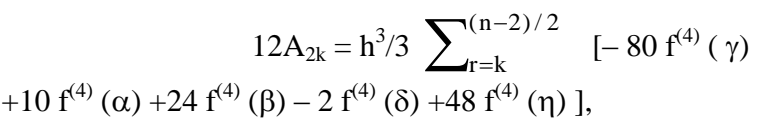

Here we use the fact that $A_{n}=0$

Therefore

$\left|\mathrm{A}_{2 \mathrm{k}}\right| \leq 41 / 18 \theta_{0} \mathrm{~h}^{2} \omega_{4}(\mathrm{~h}),\left|\theta_{0}\right| \leq 1, \mathrm{k}=0,1 \ldots,(\mathrm{n}-1) / 2$

This proves equation (L.1)

Again from equation (P.7) for $2 v \mathrm{~h} \leq \mathrm{x} \leq(2 v+1) \mathrm{h}$ we have

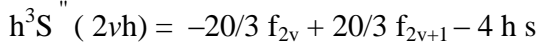

$(2 v \mathrm{~h})-$

$-8 h / 3 s^{\prime}((2 v+1) h)-h^{3} / 9 f_{2 v}{ }^{\prime \prime}+h^{2} / 3 f_{2 v+1}$

Similarly for $(2 \mathrm{v}+1) \mathrm{h} \leq \mathrm{x} \leq(2 \mathrm{v}+2) \mathrm{h}$, we obtain

(L.6) $h^{2} \mathrm{~S}^{\prime \prime}((2 \mathrm{v}+2) \mathrm{h})=-20 / 3 \mathrm{f}_{2 \mathrm{v}+2}+20 / 3 \mathrm{f}_{2 \mathrm{v}+1}+4 \mathrm{~h} \mathrm{~s}$ $(2 v+2) h)+$

$+8 \mathrm{~h} / 3 \mathrm{~s}^{\prime}((2 \mathrm{v}+1) \mathrm{h})+\mathrm{h}^{3} / 9 \mathrm{f}_{2 \mathrm{v}+2}{ }^{\prime \prime}+\mathrm{h}^{2} / 9 \mathrm{f}_{2 \mathrm{v}+1} "$.

Writing equation (L.5) for $v+1$ and subtracting from equation (L.6) .We have

$8 h A_{2 v+2}+8 h / 3 A_{2 v+1}+8 h / 3 A_{2 v+3}=-20 / 3 f_{2 v+1}+20 / 3 f_{2 v+3}-$ $2 \mathrm{~h}^{2} / 9 \mathrm{f}_{2 \mathrm{v}+2}{ }^{\prime \prime}-\mathrm{h}^{2} / 3 \mathrm{f}_{2 \mathrm{v}+1} "+$

$+h^{2} / 3 f_{2 v+3}{ }^{\prime \prime}-8 h f_{2 v+2}{ }^{\prime}-8 h / 3 f_{2 v+1}{ }^{\prime}-8 h / 3 f_{2 v+3}-8 h f_{2 v+2}{ }^{\prime}$

$-8 \mathrm{~h} / 3 \mathrm{f}_{2 \mathrm{v}+1}{ }^{\prime}-8 \mathrm{~h} / 3 \mathrm{f}_{2 \mathrm{v}+3}{ }^{\prime}$,

or

(L.7) $\quad 8 / 3\left(\mathrm{~A}_{2 \mathrm{v}+1}+\mathrm{A}_{2 \mathrm{v}+3}\right)=-8 \mathrm{~A}_{2 \mathrm{v}+2}-20 / 3 \mathrm{f}_{2 \mathrm{v}+1}+$ $20 / 3 f_{2 v+3}-2 h^{2} / 9 s^{\prime} f_{2 v+2} " '-$

$-\mathrm{h}^{2} / 3 \mathrm{f}_{2 \mathrm{v}+1}{ }^{\prime \prime}+\mathrm{h}^{2} / 3 \mathrm{f}_{2 \mathrm{v}+3}{ }^{\prime \prime}-8 \mathrm{hf}_{2 \mathrm{v}+2}-8 \mathrm{~h} / 3 \mathrm{f}_{2 \mathrm{v}+1}{ }^{\prime}-8 \mathrm{~h} / 3 \mathrm{f}_{2 \mathrm{v}+3}$ For $v=(\mathrm{n}-3) / 2, \mathrm{~A}_{\mathrm{n}}=0$. This gives

$8 / 3 A_{n-2}=-8 A_{n-1}-20 / 3 h\left[f_{n-2}-f_{n}\right]-2 h^{2} / 9 f_{n-1} " '-h / 3\left[f_{n-2}{ }^{\prime \prime}-f_{n}\right.$ $"]-8 / 3\left[3 \mathrm{f}_{\mathrm{n}-1}{ }^{\prime}+\mathrm{f}_{\mathrm{n}-2}{ }^{\prime}+\mathrm{f}_{\mathrm{n}}{ }^{\prime}\right]$, 
Using Taylor expansion for the function $\mathrm{f}$ and its derivatives on R.H.S. of this equation

We have

$8 / 3 \mathrm{~A}_{\mathrm{n}-2}=-8 \mathrm{~A}_{\mathrm{n}-1}+\mathrm{h}^{3}\left[-40 / 9 \mathrm{f}^{(4)}\left(\alpha_{1}\right)+2 / 9 \mathrm{f}^{(4)}\left(\alpha_{2}\right)-2 / 3\right.$

$\left.\mathrm{f}^{(4)}\left(\alpha_{3}\right)+4 / 3 \mathrm{f}^{(4)}\left(\alpha_{4}\right)+32 / 9 \mathrm{f}^{(4)}\left(\alpha_{5}\right)\right]$,

Therefore

$\theta_{1} \mid \leq 1$

$$
8 / 3\left|A_{n-2}\right| \leq 8\left|A_{n-1}\right|+46 / 9 \theta_{1} h^{2} \omega_{4}(h), \quad \mid
$$

Using

$\left|A_{n-1}\right| \leq 41 / 18 \theta_{0} h^{2} \omega_{4}(h)$,

we obtain

$$
\left|A_{n-2}\right| \leq 35 / 4 \theta h^{2} \omega_{4}(h),
$$

$\mid \leq 1$

Following the similar arguments for other values of $v$, we have equation(L.2) of Lemma1.

\section{Lemma 2}

Let $\mathrm{f} \in \mathrm{C}^{4}[0,1], \mathrm{n}$ any odd integer and $\mathrm{h}=1 / \mathrm{n}$. Then for $\mathrm{s}_{\mathrm{n}}(\mathrm{x})$ $=\mathrm{S}_{\mathrm{n}}(\mathrm{f}, \mathrm{x})$

ofTheorem1, we have

$$
\begin{array}{ll}
\text { (L.8) } & \left|\mathrm{s}^{\prime \prime}((2 v+1) \mathrm{h})-\mathrm{f}_{2 \mathrm{v}+1}{ }^{\prime \prime}\right| \leq \mathrm{K}_{1, \mathrm{v}} \omega_{4}(\mathrm{~h}), \\
\text { (L.9) } & \mathrm{h}\left|\mathrm{M}_{2 \mathrm{v}}-\mathrm{N}_{2 \mathrm{v}+1}\right| \leq \mathrm{K}_{2, \mathrm{v}} \omega_{4}(\mathrm{~h}),
\end{array}
$$

and

(L.10) $\quad \mathrm{h}\left|\mathrm{M}_{2 \mathrm{v}+1}-\mathrm{N}_{2 \mathrm{v}+2}\right| \leq \mathrm{K}_{3, \mathrm{v}} \omega_{4}$ (h);

where $\mathrm{K}_{1, \mathrm{v}}, \mathrm{K}_{2, \mathrm{v}}$ and $\mathrm{K}_{3, \mathrm{v}}$ are different constants depending on $v$.

\section{Proof}

From equation (P.8) we have

$h^{3} S^{\prime \prime}((2 v+1) h)=-40 f_{2 v}+40 f_{2 v+1}-12 h s^{\prime}(2 v h)-$

$-28 \mathrm{~h} \mathrm{~s}^{\prime}((2 \mathrm{v}+1) \mathrm{h})+\mathrm{h}^{3} / 3 \mathrm{f}_{2 \mathrm{v}}{ }^{\prime \prime}+8 \mathrm{~h}^{2} \mathrm{f}_{2 \mathrm{v}+1}{ }^{\prime \prime}$.

This gives on using Taylor's expansion

$\mathrm{h}^{3}\left|\mathrm{~S}^{\prime \prime}((2 \mathrm{v}+1) \mathrm{h})-\mathrm{f}_{2 \mathrm{v}+1}{ }^{\prime \prime}\right| \leq 12 \mathrm{~h}\left|\mathrm{~A}_{2 \mathrm{v}}\right|+28 \mathrm{~h}\left|\mathrm{~A}_{2 \mathrm{v}+1}\right|$ $+17 \mathrm{~h}^{4} / 3 \omega_{4}(\mathrm{~h})$

Using Lemma1 we have

| s "' $((2 v+1) \mathrm{h})-\mathrm{f}_{2 \mathrm{v}+1}{ }^{\prime \prime} \mid \leq \mathrm{K}_{1, \mathrm{v}} \omega_{4}(\mathrm{~h})$,

This proves equation (L.8).

From equation (P.9) and (P.10) we have,

$h^{4}\left(M_{2 v}-N_{2 v+1}\right)=320 f_{2 v}-320 f_{2 v+1}+120 h A_{2 v}+200 h A_{2 v+1}$

$-20 \mathrm{~h}^{3} / 3 \mathrm{f}_{2 \mathrm{v}}{ }^{\prime \prime}-$

$-40 \mathrm{~h}^{2} \mathrm{f}_{2 \mathrm{v}+1} "+120 \mathrm{~h} \mathrm{~s}^{\prime}(2 v \mathrm{~h})+200 \mathrm{~h} \mathrm{~s}{ }^{\prime}((2 v+1) \mathrm{h})$.

This can be rewritten as

$h^{4}\left(M_{2 v}-N_{2 v+1}\right)=120 h A_{2 v}+200 h A_{2 v+1}+640\left(f_{2 v}-f_{2 v+1}\right)+$ $240 \mathrm{~h} \mathrm{f}_{2 \mathrm{v}}{ }^{\prime}+$

$+400 \mathrm{hf}_{2 \mathrm{v}+1}{ }^{\prime}-40 \mathrm{~h}^{3} / 3 \mathrm{f}_{2 \mathrm{v}}{ }^{\prime \prime}-80 \mathrm{~h}^{2} \mathrm{f}_{2 \mathrm{v}+1}{ }^{\prime \prime}-\mathrm{h}^{4}\left(\mathrm{M}_{2 \mathrm{v}}-\mathrm{N}_{2 \mathrm{v}+1}\right)$,

or

$h^{4}\left(M_{2 v}-N_{2 v+1}\right)=60 h A_{2 v}+100 h A_{2 v+1}+320\left(f_{2 v}-f_{2 v+1}\right)+$

$120 \mathrm{hf}_{2 \mathrm{v}}{ }^{\prime}+$
$+200 \mathrm{hf}_{2 \mathrm{v}+1}{ }^{\prime}-20 \mathrm{~h}^{3} / 3 \mathrm{f}_{2 \mathrm{v}}{ }^{\prime \prime}-40 \mathrm{~h}^{2} \mathrm{f}_{2 \mathrm{v}+1}{ }^{\prime \prime}$.

Therefore using Taylor expansion we have

$\left|h^{4}\left(M_{2 v}-N_{2 v+1}\right)\right| \leq 60 h\left|A_{2 v}\right|+100 h\left|A_{2 v+1}\right|+100 h^{4} / 3 \omega_{4}(h)$. plugging in the values of $A_{2 v}$ and $A_{2 v+1}$ from Lemma 1, we have

$\left|\mathrm{h}^{4}\left(\mathrm{M}_{2 \mathrm{v}}-\mathrm{N}_{2 \mathrm{v}+1}\right)\right| \leq \mathrm{K}_{2, \mathrm{v}} \omega_{4}(\mathrm{~h})$.

proof for equation (L.10) can be carried out on similar lines as for (L.8) and (L.9) so we omit the details.

\section{PROOF OF THEOREM 2}

For $2 v \mathrm{~h} \leq \mathrm{x} \leq(2 \mathrm{v}+1) \mathrm{h}, v=0,1, \ldots,(\mathrm{n}-1) / 2$, we have from equation (1.5)

$$
\begin{aligned}
& \mathrm{s}^{\prime \prime}(\mathrm{x})=\mathrm{s} \text { "' }(2 v \mathrm{~h}) \mathrm{A}_{0} \\
& \frac{((2 v+1) h-x)}{h}+s^{\prime \prime \prime}((2 v+1) h) A_{0} \frac{(x-2 v h)+}{h} \\
& +\mathrm{h}^{2} \mathrm{~s}^{(5)}(2 v \mathrm{~h}) \\
& A_{1} \frac{(x-2 v h)}{h} .
\end{aligned}
$$

Now from equation (1.5) and (P.3) we have

$$
s^{(5)}(2 v h+)=-h^{-1}\left(M_{2 v}-N_{2 v+1}\right) \text {. }
$$

Since

$$
\frac{((2 v+1) h-x)}{h}+A_{0} \frac{(x-2 v h)}{h}=1,
$$

We have

$$
\begin{aligned}
& \text { (2.2) } \mathrm{s}^{\prime \prime \prime}(\mathrm{x})-\mathrm{f}^{\prime \prime \prime}(\mathrm{x})= \\
& \left(s^{\prime \prime \prime}(2 v h+)-f^{\prime \prime \prime}(x)\right) \quad A_{0} \frac{((2 v+1) h-x)}{h}+ \\
& \left(s^{\prime \prime \prime}((2 v+1) h-)-f^{\prime \prime \prime}(x)\right) \quad A_{0} \frac{(x-2 v h)}{h}- \\
& \frac{(\mathrm{x}-2 \mathrm{vh})}{\mathrm{h}} . \\
& -\mathrm{h}\left(\mathrm{M}_{2 \mathrm{v}}-\mathrm{N}_{2 \mathrm{v}+1}\right) \mathrm{A}_{1}
\end{aligned}
$$$$
=\mathrm{I}_{1}+\mathrm{I}_{2}+\mathrm{I}_{3}, \quad \text { say. }
$$

Here $\left|\mathrm{A}_{0}\right| \leq 1, \quad\left|\mathrm{~A}_{1}\right| \leq 1$.

$2 v \mathrm{~h}) \mathrm{f}^{(4)}(\alpha) \mid, 2 v \mathrm{~h} \leq \alpha \leq \mathrm{x}$

$$
\begin{aligned}
& \left|\mathrm{I}_{1}\right|=\mid\left(\mathrm{s}^{\prime \prime \prime}(2 v \mathrm{~h}+)-\mathrm{f}^{\prime \prime}(\mathrm{x}) \mid\right. \\
& =\mid\left(\mathrm{s}^{\prime \prime \prime}(2 v \mathrm{~h}+)-\mathrm{f}^{\prime \prime \prime}(2 v \mathrm{~h})+(\mathrm{x}-\right.
\end{aligned}
$$

$\Omega=\left\|\mathrm{f}^{(4)}\right\|_{\infty}$

$$
=\mid\left(\mathrm{s}^{\prime \prime \prime}(2 v \mathrm{~h}+)-\mathrm{f}^{\prime \prime \prime}(2 v \mathrm{~h}) \mid+\mathrm{h} \Omega,\right. \text { where }
$$

or

$$
\begin{aligned}
& \left|\mathrm{I}_{1}\right| \leq \mathrm{h} \Omega . \\
& \left|\mathrm{I}_{2}\right|=\left|\left(\mathrm{s}^{\prime \prime \prime}(2 \mathrm{vh}+1) \mathrm{h}-\right)-\mathrm{f}^{\prime \prime \prime}(\mathrm{x})\right|
\end{aligned}
$$


$(2 v+1) \mathrm{h}) \mathrm{f}^{(4)}(\beta) \mid$,

$$
=\mid \mathrm{s}^{\prime \prime \prime}(2 \mathrm{vh}+1)-\mathrm{f}^{\prime \prime \prime}(2 \mathrm{vh}+1)+(\mathrm{x}-
$$

Using Lemma 2, we have

$$
\left|\mathrm{I}_{2}\right| \leq \mathrm{K}_{1, \mathrm{v}} \omega_{4}(\mathrm{~h})+\mathrm{h} \Omega
$$

and

$$
\begin{aligned}
& \left|\mathrm{I}_{3}\right|=-\mathrm{h}\left(\mathrm{M}_{2 \mathrm{v}}-\mathrm{N}_{2 \mathrm{v}+1}\right) \\
& \left|\mathrm{I}_{3}\right| \leq \mathrm{K}_{2, \mathrm{v}} \omega_{4}(\mathrm{~h}) .
\end{aligned}
$$

Thus from equations $(2.2)-(2.5)$ we have the theorem for

$2 \mathrm{vh} \leq \mathrm{x} \leq(2 v+1) \mathrm{h}$ and $\mathrm{r}=3$.

Further let $(2 v+1) \mathrm{h} \leq \mathrm{x} \leq(2 v+2) \mathrm{h}, v=0,1, \ldots,(\mathrm{n}-3) / 2$.

Form equation (1.6) we have

$$
\begin{aligned}
& \mathrm{s}^{\prime \prime \prime}(\mathrm{x})=\mathrm{s}^{\prime \prime}((2 v+1) \mathrm{h}) \mathrm{A}_{0} \\
& \frac{((2 \mathrm{v}+2) \mathrm{h}-\mathrm{x})}{\mathrm{h}}+\mathrm{s}^{\prime \prime \prime}((2 \mathrm{v}+2) \mathrm{h}) \mathrm{A}_{0} \frac{(\mathrm{x}-(2 \mathrm{v}+1) \mathrm{h})}{\mathrm{h}}+ \\
& +\mathrm{h}^{2} \mathrm{~s}^{(5)}((2 \mathrm{v}+2) \mathrm{h}) \mathrm{A}_{1} \frac{((2 \mathrm{v}+2) \mathrm{h}-\mathrm{x})}{\mathrm{h}} .
\end{aligned}
$$

Using equation (1.5) and (1.3) we get

$$
\mathrm{s}^{\prime \prime \prime}(\mathrm{x})=\mathrm{s} "((2 v+1) \mathrm{h}) \mathrm{A}_{0}
$$$$
\frac{((2 v+2) h-x}{h}+s^{\prime \prime \prime}((2 v+2) h) A_{0} \frac{(x-(2 v+1) h)}{h}+
$$$$
+h\left(M_{2 v+1}-N_{2 v+2}\right) A_{1} \frac{((2 v+2) h-x)}{h} \text {. }
$$

Following similar arguments we can prove the result for

$(2 v+1) \mathrm{h} \leq \mathrm{x} \leq(2 v \mathrm{~h}+2) \mathrm{h}$ and $\mathrm{r}=3$.

Next for $r=0,1,2$, usinginterpolatory condition we can write

$$
\begin{aligned}
& \left|\mathrm{s}^{\prime \prime}(\mathrm{x})-\mathrm{f}^{\prime \prime}(\mathrm{x})\right|=\left|\int_{\mathrm{x}}^{(2 \mathrm{v}+1) \mathrm{h}}\left(\mathrm{s}^{\prime \prime \prime}(\mathrm{t})-\mathrm{f}^{\prime \prime}(\mathrm{t})\right) \mathrm{dt}\right| \\
& \leq \int_{\mathrm{x}}^{(2 \mathrm{v}+1) \mathrm{h}}\left|\mathrm{s}^{\prime \prime \prime}(\mathrm{t})-\mathrm{f}^{\prime \prime \prime}(\mathrm{t})\right| \mathrm{dt} \\
& \leq \mathrm{K}_{1, \mathrm{v}} \mathrm{h} \omega_{4}(\mathrm{~h}) .
\end{aligned}
$$

Also we can write

$\left|s^{\prime}(x)-f^{\prime}(x)\right|=\left|\int_{\lambda}^{x}\left(s^{\prime \prime}(t)-f^{\prime \prime}(t)\right) d t\right|, v h \leq \lambda \leq(v+1) h$,

Therefore,

$\left|s^{\prime}(x)-f^{\prime}(x)\right| \leq h \mid s^{\prime \prime}(t)-f^{\prime \prime}(t)$ $\leq \mathrm{K}_{1, \mathrm{v}} \mathrm{h}^{2} \omega_{4}(\mathrm{~h})$.

Similarly,

$$
\begin{aligned}
& |s(x)-f(x)|=\left|\int_{2 v h}^{x}\left(s^{\prime}(t)-f^{\prime}(t)\right) d t\right| \\
& \leq K_{1, v} h^{3} \omega_{4}(h) .
\end{aligned}
$$

This proves Theorem 2 completely.

\section{REFERENCES}

[1] Davis, P. J. Interpolation and Approximation,Blaisdell Publishing Co., New York, 1965

[2] Ahlberg, J. H. Nilson, E. N. and Walsh, J. L. The theory of Splines and their Applications, Academic Press, New York, 1967.

[3] Carl de Boor, A Practical Guide to splines, SpringerVerlag1978.

[4] Mathur, K. K. and AnjulaSaxena, Odd degree splines of higher order, Acta Math. Hung.,62 (3 - 4) (1993), 263 275 .

[5] Burkett, J. and Verma, A.K. On Birkhoff Interpolation $(0 ; 2)$ case, Aprox. Theory and its Appl. (N.S.) 11(2) (1995), 59-66.

[6] A. Saxena,SinghKulbhushan,Lacunary Interpolation by Quintic splines, Vol.66 No.1-4 (1999), 23-33, Journal of Indian Mathematical Society,

[7] Singh Kulbhushan, Interpolation by quartic splines African Jour. of Math. And Comp. Sci. Vol. 4 (10), pp.329 - 333, 15 September, 2011 ;ISSN 2006-9731

[8] Prasad, J. and Verma, A.K,.Lacunary interpolation by quintic splines SIAMJ. Numer.Anal.16,(1979) 10751079.

[9] SinghKulbhushan , Lacunary odd degree splines of higher order, Proceedings of Conference : Mathematical Science and Applications, Dec. 26-30, 2012, AbuDhabi, UAE.

[10] SaxenaAnjula, Birkhoff interpolation byquinticspline,Annales Univ. Sci. Budapest,33, (1990) 000-000.

[11] Saxena,R.B.,Lacunary Interpolation by quinticspline,SIAMJ.Numer.Anal.16,No.6 (1963) 10751079.

[12] SaxenaR.B. ,On mixed type Lacunary Interpolation II,Acta.Math.Acad.Sci.Hung.14,(1963)1-19.

[13] SaxenaR.B. and Joshi T.C., On quartic spline Interpolation Ganita 33, No. 2, (1982)97-111.

[14] SallamS.On interpolation by quintic Spline, Bull. Fac.Sci.Assiiut.Univ,11(1), (1982) 97- 106. 